\title{
FRAMEWORK PARA ESCOLHA DAS ESTRATÉGIAS DE APRENDIZAGEM ATIVA: APLICAÇÃO NA DISCIPLINA DE METODOLOGIA DA PESQUISA CIENTÍFICA E TECNOLÓGICA NUM CURSO DE ENGENHARIA DE PRODUÇÃO
}

Denise Luciana Rieg - denise.rieg@maua.br

Instituto Mauá de Tecnologia, Departamento de Engenharia de Produção

Praça Mauá 1

09580-900 - São Caetano do Sul - SP

Rui Manuel Lima - rml@dps.uminho.pt

Universidade do Minho, Departamento de Produção e Sistemas

Campus de Azurém

4800-058 - Guimarães, Portugal

Fernando C. L. Scramim-fernando.scramim@maua.br

Instituto Mauá de Tecnologia

Praça Mauá 1

09580-900 - São Caetano do Sul - SP

Octavio Mattasoglio Neto-omattasoglio@maua.br

Instituto Mauá de Tecnologia, Ciclo Básico

Praça Mauá 1

09580-900 - São Caetano do Sul - SP

Resumo: $O$ objetivo do presente trabalho é propor um framework de análise que auxilie o professor na escolha da estratégia de ensino para o desenvolvimento de competências técnicas e transversais. Complementando a proposta, exemplifica-se o uso do referido framework na escolha do método de aprendizagem ativa a ser utilizado na disciplina de Metodologia da Pesquisa Científica e Tecnológica de um curso de Engenharia de Produção, ao ministrar um dos tópicos do seu ementário. Os métodos de pesquisa utilizado no desenvolvimento deste trabalho foram o estudo de caso e o survey. Este último se fez necessário pelo fato de o framework proposto ter como um de seus passos uma pesquisa junto aos alunos para identificar suas preferências em relação às estratégias de aprendizagem ativa.

Palavras-chave: Aprendizagem ativa. Framework. Competências Técnicas. Competências transversais. 


\section{INTRODUÇÃO}

Os engenheiros, no cenário, atual devem ser capazes não só de propor soluções tecnicamente corretas para os problemas, mas de tratá-los em sua totalidade, conectados a causas e efeitos de múltiplas dimensões. Aliado a isto, é demandado dos novos engenheiros, a capacidade de coordenar informações, de trabalhar em grupo, liderar pessoas, interpretar a realidade de forma dinâmica e aprender de modo autônomo e ao longo da vida (MEC, 2019). São as chamadas soft skills (competências transversais).

No sentido de impulsionar os cursos de Engenharia no país a proporcionarem a formação com foco no ensino por competências, as novas Diretrizes Curriculares Nacionais do Curso de Graduação em Engenharia (DCNs de Engenharia) (MEC, 2019), declaram a necessidade de empregar ferramentas de ensino mais ativas, colocando o aluno como peça-chave na busca por conhecimento, envolvendo-o de forma mais contundente no processo de aprendizagem.

O espectro dos modelos de aprendizagem ativa é amplo, indo do emprego de métodos mais centrados no professor associados às aulas expositivas, como brainstorming e estudos de casos, ao emprego de métodos mais centrados nos alunos, como o aprendizado baseado em projetos (Project-based learning) (LORD et al., 2012).

Apesar de serem todos modos indutivos de ensino e aprendizagem que buscam engajar os alunos no processo de aquisição de conhecimento, cada método tem suas especificidades e contribui de forma particular para o desenvolvimento de competências técnicas e transversais (PRINCE; FELDER, 2006). A escolha dentre eles, portanto, depende, primordialmente, do objetivo da aprendizagem, das competências que se quer desenvolver. Prince e Felder (2007) colocam que além do objetivo da aprendizagem, a escolha deve ser pautada também pela existência ou não dos recursos necessários à aplicação do método e na experiência e tempo disponível do professor para sua aplicação, que muitas vezes exige extensivo acompanhamento do que é desenvolvido pelos alunos.

Estes fatores, sem dúvida, são essenciais na escolha do método a ser empregado pelo professor no planejamento de suas aulas. Mas há algum outro aspecto a ser também levado em consideração para aumentar as chances de proficuidade do processo de aprendizagem?

Neste sentido, o objetivo do presente trabalho é propor um framework de análise que auxilie o professor na escolha do método de aprendizagem para o desenvolvimento de competências técnicas e transversais. Complementando a proposta, exemplifica-se o uso do referido framework na escolha do método de aprendizagem ativa a ser utilizado na disciplina de Metodologia da Pesquisa Científica e Tecnológica (MPCT) do Curso de Engenharia de Produção do Instituto Mauá de Tecnologia (IMT), ao ministrar um dos tópicos do seu ementário.

Com tal propósito, na seção 2, são descritos alguns dos métodos de aprendizagem ativa mais usuais em engenharia, bem como, exemplos da contribuição dos mesmos para o alcance de competências, conforme já expostos na literatura. O framework proposto é apresentado na seção 3. Na seção 4, tem-se a descrição do método de pesquisa e, na seção 5, a exemplificação do uso do framework. A seção 6, apresenta as considerações finais desse artigo.

\section{REVISÃO BIBLIOGRÁFICA}

Aprendizagem ativa é uma estratégia de ensino que busca promover maior engajamento do estudante no processo de aprendizagem. Dentro dessa estratégia, existe uma grande variedade de métodos ou práticas instrucionais. Alguns já pressupõem o trabalho em grupo, a ação cooperativa entre os alunos ou a competitividade entre eles. Outros, permitem a escolha de 
modalidade, se individual ou em grupo, se cooperativa ou competitiva. São ainda passíveis de adaptações, conforme o professor estabelece sua prática, sem perder a essência do método.

São apresentados, a seguir, alguns desses métodos de aprendizagem ativa e como os mesmos podem contribuir para o desenvolvimento de habilidades e competências, sem a pretensão de esgotar todas as suas possibilidades.

$\mathrm{Na}$ Aprendizagem Baseada em Problemas (Problem-based learning - PBL) e na aprendizagem Baseada em Projetos (Project-based learning - PjBL), os alunos devem compreender o contexto e as especificidades do problema ou projeto, estabelecer metas, alocar recursos apropriados e determinar a metodologia a ser utilizada para abordar o problema/projeto (LORD et al., 2012). O professor, por sua vez, assume o papel de mediador do processo de aprendizagem, estimulando discussões sobre os assuntos em pauta e a participação de todos, assistindo os estudantes, planejando ambientes de aprendizagem instigantes e avaliando o desempenho e a aprendizagem dos discentes, dando-lhes feedbacks constantes (ELMOR FILHO et al., 2019). Enquanto o PBL tem como foco um problema contextualizado e pouco estruturado, o PjBL tem como foco um projeto, sendo um dos principais objetivos relacionar conteúdos interdisciplinares de forma integrada. Essencialmente, em ambos os casos, os trabalhos são desenvolvidos em grupo pelos alunos (LORD et al., 2012). O PBL e o PjBL são métodos apropriados para desenvolver competências como trabalhar em grupo, liderar equipes, aprender de modo autônomo e ao longo da vida, formular e conceber soluções desejáveis de Engenharia, projetar e analisar sistemas, produtos e serviços, componentes ou processos (PRINCE; FELDER, 2006).

A Aprendizagem Baseada em Pesquisa (Research-based learning - RBL) tem como propósito fazer com que os alunos projetem e vivenciem o processo de uma pesquisa no âmbito de sua formação. Os alunos identificam um problema, revisam a literatura acerca do tema, formam hipóteses, desenvolvem o protocolo de pesquisa, coletam e analisam os dados, discutem os resultados e formulam conclusões. Desta forma, o RBL é um método que permite adquirir e sustentar conhecimento acerca de um determinado assunto, desenvolver pensamento crítico (que inclui interpretação, análise, avaliação, inferência e explicação acerca do tema tratado) e conceber soluções para problemas em análise, na mesma linha dos métodos PBL e PjBL. Contribui também para melhorar a capacidade de trabalho em equipe, de comunicação e de aprender de forma autônoma (SUSIANI; SALIMI; HIDAYAH, 2018; SINGH et al., 2019).

Por meio da Sala de Aula Invertida (flipped classroom), os alunos absorvem o conteúdo disponibilizado pelo professor por meio virtual (vídeos, áudios, podcast, textos, etc.) antes da aula para que os mesmos estejam cientes dos assuntos a serem tratados em classe. Na sala de aula, há a interação professor-aluno, para sanar dúvidas, discutir e desenvolver o assunto. Assim, esse método faz uso intensivo das Tecnologias de Informação e Comunicação para transmitir os conceitos básicos aos alunos, permitindo que o tempo em sala de aula seja utilizado para a realização de atividades mais interativas e que exijam dos alunos processos de raciocínio mais complexos (SUHR, 2016). É um método bastante útil quando se pretende usar o tempo em sala de aula para desenvolver "[...] habilidades do tipo interpretação de dados, design, formulação e resolução de problemas, comunicação, pensamento crítico, trabalho em equipe, dentre outras [...]" (ELMOR FILHO et al., 2019, p. 45). O nível, entretanto, do desenvolvimento de tais habilidades depende do proposto pelo professor em sala de aula e não simplesmente e intrinsecamente pela inversão que caracteriza o método. Burgett (2014) ressalta que o sucesso da sala de aula invertida é, antes de tudo, dependente da motivação dos alunos para acessar extraclasse o material disponibilizado previamente pelo professor e que a exposição prévia de material é útil quando o conteúdo a ser assimilado não é complexo.

A Instrução pelos Colegas (Peer Instruction - PI), em um primeiro momento, solicita que os alunos busquem informações primárias sobre um determinado assunto por meio de outras 
fontes que não seja o professor (textos, vídeos, etc.). A busca de informações pode se dar antes ou na própria aula. Após essa etapa, o professor conduz uma curta exposição dialogada e apresenta uma questão conceitual que deve ser respondida individualmente pelos alunos. É usual a utilização de sistemas de votação como Kahoot ou Socrative para que as respostas dos alunos sejam dadas e vistas pelo professor. Mediante o grau de acerto, o professor revisita o conceito em pauta, promove discussão em classe ou passa a tratar outro assunto (ELMOR FILHO et al., 2019). Esse método é útil para promover um ambiente de cooperação entre os alunos e que colaborem no processo de aprendizagem. Segundo Gok e Gok (2017), relativamente aos resultados do Peer Instruction relatado por outros pesquisadores, o método auxilia no desenvolvimento do pensamento crítico e na capacidade de tomada de decisão.

O método Pensar, Compartilhar e Socializar (Think-Pair-Share - TPS) demanda inicialmente que os alunos desenvolvam ideias individuais sobre o tema em questão (Pensar) para, num segundo momento, apresentar e discutir em pares suas percepções (Compartilhar). Finalizando o processo, na fase do socializar, os pares de alunos compartilham suas ideias. Como o Peer Instruction, o TPS promove um ambiente de cooperação, comunicação e compartilhamento de conhecimento entre pares (DESHPANDE; SALMAN, 2016).

O Estudo de Caso (EC) é um método de aprendizagem ativa que coloca os alunos em contato com casos relacionados com sua área de formação profissional, que apresentem um problema mal estruturado, exigindo empenho dos mesmos para identificar e analisar o problema, avaliar e propor soluções, pensar criticamente e tomar decisões. (HARPER; LAMB; BUFFINNGTON, 2008). Segundo em Elmor Filho et al. (2019, p. 114), o EC "exige uma grande dedicação do estudante na preparação da resolução do caso, levantando hipóteses e explicações, bem como reunindo evidências que o comprovem. O EC também demanda dos estudantes a capacidade de trabalhar em grupos".

A simulação propõe a experimentação, a imitação de alguns aspectos da realidade, permitindo que os alunos desenvolvam seus conhecimentos. É um método que permite criar experiências mais realistas de aprendizagem (ANWAR; SAMI; HAKIM, 2018). Pode ou não fazer uso de modelos matemáticos e computacionais. De acordo com Qieni, Yi e Hongbin (2017) a simulação é um método que estimula os alunos a questionar, interpretar dados e situações, pensar criticamente e, em muitos casos, exige a formulação e resolução de problemas.

Geração de Ideias (brainstorming) é a contribuição espontânea de ideias dos alunos para buscar a solução de um problema, explorar ideias e conceitos ou criar algo novo. Como exposto em Lim, Yusof e Ismail (2018), esse método é útil para estimular o pensamento criativo, identificar possíveis soluções e selecionar a melhor opção para problemas em análise.

A Gamificação é a realização de jogos por equipes ou indivíduos, de competição ou colaboração, em diversas áreas de conhecimento, com o propósito de engajar os alunos na resolução de problemas, motivar ações e melhorar o aprendizado. Em termos de desenvolvimento de competências, Santos-Souza e Oliveira (2019) colocam que os jogos de empresas permitem que os alunos vivenciem a tomada de decisão sob risco e incerteza, estabeleçam relações de causa e efeito e desenvolvam competências gerenciais em um processo de aprender por fazer. A gamificação (Quizzes) tem sido utilizada como forma de avaliação e feedback imediato aos alunos após exposição e discussão acerca de um tema (CAVADAS et al., 2017).

\section{FRAMEWORK PARA ESCOLHA DAS ESTRATÉGIAS DE APRENDIZAGEM ATIVA}

O framework de análise ora proposto tem por objetivo ser uma ferramenta que auxilie o professor na escolha do método de aprendizagem no planejamento de suas aulas. A base são os 
fatores apontados por Prince e Felder (2007) para tal propósito - objetivo da aprendizagem, disponibilidade de recursos e de tempo do professor para a aplicação do método.

Acrescenta-se a essa sequência de análise as competências transversais a serem desenvolvidas e a percepção dos alunos quanto às alternativas possíveis, ou seja, a identificação dos métodos que consideram atrativos para promover seu aprendizado. O propósito é estimulálos ainda mais para o processo de aprendizagem, promover nos mesmos uma postura positiva durante as aulas e extraclasse favorecendo a aquisição de conhecimentos e o desenvolvimento de competências. Não se trata de transferir aos alunos pura e simplesmente a escolha do método a ser adotado, mas dado o conjunto de métodos que atendam os propósitos de capacitação desejados e tenham condições efetivas de serem implementados, que se inclua a apreciação dos alunos no processo de escolha. A Figura 1 apresenta o framework de análise proposto.

Figura 1 - Framework de análise para seleção de métodos de aprendizagem ativa

\begin{tabular}{|c|c|c|}
\hline $\begin{array}{c}\text { 1. Quais são os objetivos } \\
\text { de aprendizagem? }\end{array}$ & $\begin{array}{c}\text { Métodos de aprendizagem } \\
\text { ativa/modalidade }\end{array}$ & $\begin{array}{c}\text { Objetivos de aprendizagem: lembrar, } \\
\text { compreender, aplicar, analisar, avaliar, } \\
\text { projetar, implantar, supervisionar e controlar } \\
\text { soluções, etc. }\end{array}$ \\
\hline $\begin{array}{l}\text { 2. Quais são as } \\
\text { competências transversais } \\
\text { a serem desenvolvidas? }\end{array}$ & $\begin{array}{l}\text { cooperativa ou } \\
\text { competitivas) }\end{array}$ & $\begin{array}{c}\text { Competências transversais: coordenar } \\
\text { informações, trabalhar em grupo, liderar } \\
\text { pessoas, aprender ao longo da vida (lifelong } \\
\text { learning), comunicar-se, etc. }\end{array}$ \\
\hline $\begin{array}{l}\text { 3. Quais são os recursos } \\
\text { necessários para a } \\
\text { implementação do método? }\end{array}$ & & $\begin{array}{c}\text { Recursos: tempo; tecnologias de informação e } \\
\text { comunicação (TICs), instalações físicas, } \\
\text { softwares, etc. }\end{array}$ \\
\hline $\begin{array}{l}\text { 4. Quais métodos são } \\
\text { considerados atrativos } \\
\text { pelos alunos para promover } \\
\text { seu aprendizado? }\end{array}$ & & $\begin{array}{l}\text { Pesquisa com os alunos (survey) sobre } \\
\text { preferências em relação aos métodos de } \\
\text { aprendizagem ativa. }\end{array}$ \\
\hline
\end{tabular}

Seguindo o proposto no framework, primeiramente analisam-se os métodos de aprendizagem ativa quanto à possibilidade de atingir os objetivos de aprendizagem da disciplina ou do tópico específico a ser ministrado. Os métodos que atendam os objetivos traçados são analisados quanto à sua contribuição para o alcance das competências transversais desejadas. Nesse momento, avalia-se também a possibilidade de modalidade de aplicação dos métodos, se individual ou em grupo, se de forma cooperativa ou competitiva. Os métodos que passarem também por esse crivo são avaliados quanto aos recursos necessários para sua implementação, se os recursos estão ou não disponíveis, incluindo o tempo de dedicação do professor para sua aplicação. O conhecimento e a experiência do professor com os métodos sob análise contribuem significativamente para a avaliação dos mesmos.

Por fim, o conjunto de métodos que tenham passado até este ponto do funil de análise, são avaliados quanto à apreciação dos alunos no que tange à motivação que promovem para seu aprendizado, o que eles consideram como sendo os métodos mais atrativos de aprendizagem ativa, que instigam sua participação e interesse no processo (realização de um survey). Cabe notar que, relativamente à pesquisa com os alunos, quanto mais familiarizados com os tipos de aprendizagem ativa estiverem (assim como no caso dos professores), mais eficaz será sua participação na fase de escolha do método. De qualquer forma, na pesquisa junto aos alunos é necessário explicar como funciona cada método que inclui o roll de possibilidades. 


\section{MÉTODO DE PESQUISA}

Para exemplificar o uso do framework aqui proposto foi realizado um estudo de caso na disciplina de MPCT ministrada no quarto ano de Engenharia de Produção do Instituto Mauá de Tecnologia - IMT. A disciplina tem por objetivo levar os alunos a refletir e discutir sobre os pressupostos, objetivos e características do trabalho científico e tecnológico e suas implicações na vida acadêmica e empresarial.

Pretende-se, em termos de competências técnicas, que os alunos, ao cursar esta disciplina, sejam capazes de estruturar a construção de uma pesquisa científica considerando suas diversas fases (identificação do objetivo; elaboração do projeto; condução da investigação; interpretação dos resultados; e escolha dos instrumentos adequados para a divulgação dos resultados) e considerando as normas para elaboração de trabalhos acadêmicos, conforme ABNT Associação Brasileira de Normas Técnicas. Em relação às competências transversais a disciplina pretende contribuir principalmente para aprimorar a capacidade de coordenar informações, de pesquisa, de comunicação dos alunos na forma escrita e oral, além de aprimorar a capacidade de trabalho em grupo.

Atualmente a disciplina se divide em dois momentos: o primeiro semestre volta-se para a exposição e discussão dos conteúdos essenciais à elaboração de trabalhos científicos e o segundo semestre volta-se para a elaboração do projeto de trabalho de conclusão de curso (TCC) a ser desenvolvido no ano seguinte.

Com base em um tópico específico a ser ministrado na disciplina, percorreu-se o funil de análise presente no framework proposto na seção 3. Como o último passo do mesmo prevê a realização de pesquisa junto aos alunos para identificar suas preferências em relação aos métodos de aprendizagem ativa, foi realizado, um levantamento amostral (survey) com 111 alunos da disciplina de MPCT, turmas de 2019, dos 134 alunos matriculados. O survey foi conduzido por meio de questionário, no qual foi solicitado aos alunos que assinalassem os métodos de aprendizagem ativa (sem restrição de número) que consideravam atrativos para promover o seu aprendizado. Havia no questionário a explicação de cada um dos métodos.

\section{RESULTADOS}

O tópico da disciplina MPCT escolhido para exemplificar o uso do framework proposto neste artigo foi "Técnicas de coleta de dados" utilizado na condução de estudos de casos, pesquisa-ação, modelagem e simulação e surveys, métodos mais usuais no âmbito da Engenharia de Produção. Nesses métodos de pesquisa, as fontes de coletas de dados mais utilizadas são entrevistas, análise documental, observação in loco/in modus operandi e questionários, sendo recomendado sempre que possível, a utilização de múltiplas fontes de evidências. O objetivo de aprendizagem é compreender diferentes formas de coletas de dados, analisar dados e aplicar a técnica de triangulação de dados. Em relação as competências transversais, o foco era a capacidade de coordenar informações, trabalhar em grupo e se comunicar de forma escrita. O Quadro 1 cruza os objetivos e competências com os métodos de aprendizagem ativa, destacando as contribuições que podem promover, percorrendo as duas primeiras fases do framework.

Do Quadro 1, PBL, PjBL, RBL, Flipped classroom, Estudo de Caso, Simulação e Gamificação são métodos que, cada um a seu modo, permitem que os objetivos de aprendizagem propostos (compreender, analisar e aplicar) sejam alcançados e contribuem para o desenvolvimento das competências transversais desejadas. Especificamente em relação ao método Flipped classroom, sua adequação aos objetivos de aprendizagem e às competências é dependente de como os conhecimentos adquiridos por meio virtual previamente às aula são 
trabalhados em classe. Peer Instruction (PI) e Think-Pair-Share (TPS), por outro lado, são mais adequados à reflexão compartilhada e ao desenvolvimento do pensamento crítico do que à aplicação de conhecimentos e ferramentas, como também pretendido. Da mesma forma, Brainstorming não se adequa aos objetivos de aprendizagem traçados, sendo um método útil para compreender e explorar ideias.

Quadro 1 - Objetivos de aprendizagem e competências transversais versus métodos de aprendizagem ativa

\begin{tabular}{|l|c|c|c|c|c|c|c|c|c|c|}
\hline $\begin{array}{c}\text { Objetivos de Aprendizagem e } \\
\text { Competências transversais }\end{array}$ & $\mathrm{PBL}$ & $\mathrm{PjBL}$ & $\mathrm{RBL}$ & $\mathrm{FC}$ & $\mathrm{PI}$ & $\mathrm{TPS}$ & $\mathrm{EC}$ & $\mathrm{S}$ & $\mathrm{B}$ & $\mathrm{G}$ \\
\hline Compreender & $\mathrm{X}$ & $\mathrm{X}$ & $\mathrm{X}$ & $\mathrm{X}$ & $\mathrm{X}$ & $\mathrm{X}$ & $\mathrm{X}$ & $\mathrm{X}$ & $\mathrm{X}$ & $\mathrm{X}$ \\
\hline Aplicar & $\mathrm{X}$ & $\mathrm{X}$ & $\mathrm{X}$ & $\mathrm{X}$ & & & $\mathrm{X}$ & $\mathrm{x}$ & & $\mathrm{x}$ \\
\hline Analisar / interpretar dados & $\mathrm{X}$ & $\mathrm{X}$ & $\mathrm{X}$ & $\mathrm{X}$ & $\mathrm{X}$ & $\mathrm{X}$ & $\mathrm{X}$ & $\mathrm{X}$ & & $\mathrm{X}$ \\
\hline Trabalhar em grupo & $\mathrm{X}$ & $\mathrm{X}$ & $\mathrm{X}$ & $\mathrm{X}$ & $\mathrm{X}$ & $\mathrm{X}$ & $\mathrm{X}$ & $\mathrm{X}$ & $\mathrm{x}$ & $\mathrm{X}$ \\
\hline Coordenar informações & $\mathrm{X}$ & $\mathrm{X}$ & $\mathrm{X}$ & $\mathrm{x}$ & & & $\mathrm{X}$ & $\mathrm{X}$ & & $\mathrm{X}$ \\
\hline $\begin{array}{l}\text { Comunicar-se de forma escrita, } \\
\text { oral e gráfica }\end{array}$ & $\mathrm{X}$ & $\mathrm{X}$ & $\mathrm{X}$ & $\mathrm{X}$ & $\mathrm{X}$ & $\mathrm{X}$ & $\mathrm{x}$ & $\mathrm{x}$ & & $\mathrm{x}$ \\
\hline
\end{tabular}

Legenda: Problem-based learning (PBL); Project-based learning (PjBL); Research-based learning (RBL); Flipped classroom (FC); Peer Instruction (PI); Think-Pair-Share (TPS); Estudo de Caso (EC); Simulação (S); Brainstorming (B); e Gamificação (G); X: maior contribuição; $x$ : menor contribuição.

Fonte: Autores (2020).

Quando a análise se voltou para os recursos disponíveis, o principal limitador foi o recurso "tempo". Como exposto na seção anterior, o primeiro semestre da disciplina de MPCT volta-se para a exposição e discussão dos conteúdos essenciais à elaboração de trabalhos científicos, para que, no segundo semestre, os alunos possam, por meio de um processo mais autônomo, desenvolver seus projetos de TCC. Assim, na primeira parte da disciplina, pretende-se que os alunos tenham, paulatinamente, contato com os diferentes tópicos que constituem a sua ementa e em uma sequência coerente que alinhe as partes principais de um trabalho científico, de forma que cada conhecimento adquirido seja base para compreender o próximo tópico a ser discutido. Dessa forma, a disciplina, na sua primeira fase, busca sim envolver o aluno de forma contundente no processo de aprendizagem, mas passo a passo na busca do conhecimento, distribuindo-se os tópicos dentro de uma programação aula-a-aula. Isto não quer dizer que, uma vez ministrado o tópico, o mesmo não seja mais abordado, pelo contrário, retomar o que já foi discutido é essencial para se construir o alinhamento desejado. Entretanto, tem-se aulas específicas para se iniciar cada novo assunto. Assim, dispunha-se, à princípio, de 4 horas-aula para conhecer as diferentes formas de coleta de dados, aplicar a técnica de triangulação de dados e experienciar o processo de análise de dados.

Frente a limitação deste recurso, foram descartados os métodos PBL, PjBL e RBL, por demandarem mais tempo para a sua execução. De fato, são métodos mais utilizados quando os objetivos centrais são promover a aprendizagem de modo autônomo e ao longo da vida e formular e conceber soluções desejáveis para os problemas em análise, como já destacado aqui, o que requer mais tempo de laboração. A gamificação também foi descartada pelo professor, por não dispor de recursos dessa natureza que permitisse alcançar os objetivos propostos.

Assim, restaram para a última etapa os métodos Simulação, Flipped Classroom e Estudo de Caso. Complementando a análise proposta pelo framework, tem-se o resultado do survey realizado com os alunos da disciplina de MPCT quanto às suas preferências em relação aos métodos de aprendizagem ativa, como exposto na Tabela 1.

O método Flipped Classroom que, inclusive, poderia ser combinado com o Estudo de caso ou a Simulação, foi descartado pois teve aprovação de apenas 15,3\% e sabe-se que seu sucesso depende da motivação dos alunos para acessar extraclasse o material disponibilizado previamente pelo professor. 
Por fim, entre Estudo de Caso e Simulação, este último foi escolhido por ter maior aceitação dos alunos, embora tenha apresentado pequena vantagem em termos de preferência dos alunos quando comparado com o método de estudo de caso.

Figura 2 - Exemplo da utilização do framework de análise para seleção de métodos de aprendizagem ativa

\begin{tabular}{|c|c|c|}
\hline $\begin{array}{c}\text { 1. Quais são os objetivos } \\
\text { de aprendizagem? }\end{array}$ & $\begin{array}{c}\text { PBL, PjBL, RBL, Flipped } \\
\text { classroom, Estudo de Caso, } \\
\text { Simulação } e \text { Gamificação }\end{array}$ & $\begin{array}{l}\text { Objetivos: compreender diferentes formas de } \\
\text { coletas de dados (observação in loco, análise } \\
\text { de documentos, entrevistas e questionários), } \\
\text { analisar dados coletados e aplicar a técnica de } \\
\text { triangulacão de dados. }\end{array}$ \\
\hline $\begin{array}{l}\text { 2. Quais são as } \\
\text { competências transversais } \\
\text { a serem desenvolvidas? }\end{array}$ & $\begin{array}{l}\text { Flipped classroom, } \\
\text { Estudo de Caso, } \\
\text { Simulação } e \\
\text { Gamificação }\end{array}$ & $\begin{array}{l}\text { Competências transversais: coordenar } \\
\text { informações, trabalhar em grupo e } \\
\text { comunicar-se de forma escrita, etc. }\end{array}$ \\
\hline $\begin{array}{l}\text { 3. Quais são os recursos } \\
\text { necessários para a } \\
\text { implementação do método? }\end{array}$ & $\begin{array}{l}\text { Simulação } e \\
\text { Gamificaçã }\end{array}$ & $\begin{array}{l}\text { Recursos: tempo (4 horas aulas); Kahoot, } \\
\text { salas de aprendizagem ativa própria para } \\
\text { trabalhos em grupo, artigos científicos acerca } \\
\text { do tema, Excel, Word e vídeos. }\end{array}$ \\
\hline $\begin{array}{l}\text { 4. Quais métodos são } \\
\text { considerados atrativos } \\
\text { pelos alunos para promover } \\
\text { seu aprendizado? }\end{array}$ & $\begin{array}{l}\text { Es fudo de Cho, } \\
\text { Flippled classiogm }\end{array}$ & $\begin{array}{l}\text { Survey: simulação (70,4\%), estudo de caso: } \\
\quad 60,3 \% \text { e flipped classroom }(15,3 \%)\end{array}$ \\
\hline
\end{tabular}

Tabela 1 - Estratégias de aprendizagem ativa consideradas atrativas pelos alunos

\begin{tabular}{|l|c|}
\hline \multicolumn{1}{|c|}{ Tipo de metodologia de aprendizagem ativa } & Porcentagem de alunos \\
\hline Aprendizagem Baseada em Problemas ou problem-based learning & $71,2 \%$ \\
\hline Aprendizagem Baseada em Projetos ou project-based learning & $60,7 \%$ \\
\hline Aprendizagem Baseada em Pesquisa ou Research-based learning & $24,3 \%$ \\
\hline Instrução pelos Colegas (Peer Instruction) & $15,3 \%$ \\
\hline Sala de Aula Invertida (Flipped Classroom) & $15,3 \%$ \\
\hline Simulação & $70,4 \%$ \\
\hline Pensar, Compartilhar e Socializar (Think-Pair-Share - TPS) & $29,7 \%$ \\
\hline Estudo de Casos & $60,3 \%$ \\
\hline Geração de Ideias (brainstorming) & $59,5 \%$ \\
\hline Gamificação & $42,3 \%$ \\
\hline
\end{tabular}

Fonte: Autores (2019).

\section{CONSIDERAÇÕES FINAIS}

O presente artigo apresentou um framework para seleção de métodos de aprendizagem ativa que inclui, a análise dos objetivos de aprendizagem, das competências transversais a se desenvolver, dos recursos disponíveis e da percepção dos alunos sobre esses métodos, no que tange à atratividade para promover seu aprendizado. Ao incluir esse último passo na escolha do método, a proposta é aumentar as chances de proficuidade do processo de aprendizagem, ao entender que os métodos de aprendizagem ativa não estimulam da mesma forma todos os estudantes a adquirir o conhecimento pretendido. E promover efetivamente a propensão dos alunos para a aprendizagem dentro e fora da sala de aula é papel essencial do educador.

A oportunidade de os estudantes participarem da escolha do processo de aprendizagem faz com que se tornem colaboradores da estruturação da aula de forma mais ampla, da decisão do professor de como conduzir o processo ensino-aprendizagem. O professor conduz o processo que é determinado por todos, até mesmo os alunos. Essa responsabilidade da à flexibilidade, 
que se busca na construção de currículos atuais, uma nova dimensão que é a escolha do estudante, para além da infraestrutura, tempo e conhecimento do professor.

\section{REFERÊNCIAS}

ANWAR, M.; SAMI, H.; HAKIM, A. Teaching Lean Manufacturing in Educational Field Through LBD: A Case Study in an Engineering School. International Journal of Vocational Education and Training Research, 4(1), PP. 41-44, 2018.

BURGETT, J. Can "Flipping the Classroom" Improve the Educational Experience in Construction Management Courses: a case study in teaching HVAC psychrometrics. In: PROCEEDINGS OF THE 50TH ASC ANNUAL INTERNATIONAL CONFERENCE, COLLEGE STATION, TX, 2014.

CAVADAS, C.; GODINHO, W.; MACHADO, C. T.; CARVALHO, A. A. Quizzes as an active learning strategy: A study with students of pharmaceutical sciences. In:12th IBERIAN CONFERENCE ON INFORMATION SYSTEMS AND TECHNOLOGIES(CISTI), 2017.

DESHPANDE, A.; SALMAN, B. Application of an Active Learning Technique in Engineering and Construction Management Classes. In: 52ND ASC ANNUAL INTERNATIONAL CONFERENCE PROCEEDINGS, 2016.

ELMOR FILHO, G.; SAUER, L. Z.; ALMEIDA, N. N. D.; VILLAS-BOAS, V. Uma nova sala de aula é possível: aprendizagem ativa na educação em engenharia. 1 ed. Ruo de Janeiro: LTC, 2019.

GOK, T. GOK, O. Peer instruction: an evaluation of its theory, application, and contribution. Asia-Pacific Forum on Science Learning and Teaching, 18(2), p.2, 2017.

HARPER, J. S.; LAMB, S. W.; BUFFINGTON, J. R. Effective Use of Case Studies in the MIS Capstone Course through Semi-Formal Collaborative Teaching. Journal of Information Systems Education, 19(4), 2008.

LIM, K. K., YUSOF, Y. M., \& ISMAIL, Z. Creative Thinking of Engineering Undergraduates through Brainstorming during Mathematical Problem Solving. In: IEEE INTERNATIONAL CONFERENCE ON TEACHING, ASSESSMENT, AND LEARNING FOR ENGINEERING (TALE), 2018.

LORD, S. M.; PRINCE, M. J.; STEFANOU, C. R.; STOLK, J. D.; CHEN, J. C. The effect of different active learning environments on student outcomes related to life long learning. International Journal of Engineering Education, 28(3), pp. 606-620, 2012.

MEC - Ministério da Educação, Resolução no 2, de 24 de abril de 2019. Diretrizes Curriculares Nacionais do Curso de Graduação em Engenharia. Parecer Homologado Despacho do Ministro, publicado no D.O.U. de 23/4/2019, Seção 1, p. 109. Disponível em: http://portal.mec.gov.br/index.php?option=com_docman\&view=download\&alias=109871pces001-19-1\&category_slug=marco-2019-pdf\&Itemid=30192. Acesso em: 29/09/2019. 
PRINCE, M.; FELDER R. M. Inductive Teaching and Learning Methods: definitions, comparisons, and research bases. Journal of Engineering Education, 95(2), pp. 123-38, 2006.

PRINCE, M.; FELDER R. M. The Many Faces of Inductive Teachingand Learning. Journal of College Science Teaching, 36(5), pp. 14-20, 2007.

SANTOS-SOUZA, H. R. D.; OLIVEIRA, M. A. O Uso de Jogos de Empresas em Diferentes Níveis Educacionais: Integração, Prática e Pesquisa Envolvendo Estudantes de Graduação e Pós-Graduação em Administração. Administração: Ensino e Pesquisa (RAEP), 20(1), 2019.

SILVA, J. B. D.; SALES, G. L.; CASTRO, J. B. D. Gamificação como estratégia de aprendizagem ativa no ensino de Física. Revista Brasileira de Ensino de Física, 41(4), 2019.

SINGH, R.; DEVIKA; HERRMANN, C.; THIEDE, S.; SANGWAN, K. S. Research-based Learning for Skill Development of Engineering Graduates: An empirical study. Procedia Manufacturing, 31, pp. 323-329, 2019.

SUHR, I. R. F. Desafios no uso da sala de aula invertida no ensino superior. R. Transmutare, Curitiba, 1(1), p. 4-21, 2016.

SUSIANI, T. S.; SALIMI, M.; HIDAYAH, R. Research Based Learning (RBL): How to Improve Critical Thinking Skills? In: SHS WEB OF CONFERENCES, 42, 00042, 2018.

QIENI, L.; YI, W. M.D.; HONGBIN L. Simulation teaching method in Engineering Optics. In: 14TH CONFERENCE ON EDUCATION AND TRAINING IN OPTICS AND PHOTONICS, ETOP HANGZHOU, CHINA, 2017.

\title{
FRAMEWORK FOR CHOOSING ACTIVE LEARNING STRATEGIES: APPLICATION IN THE SCIENTIFIC AND TECHNOLOGICAL RESEARCH METHODOLOGY DISCIPLINE IN A INDUSTRIAL ENGINEERING COURSE
}

\begin{abstract}
The objective of this work is to propose an analysis framework that helps the teacher in choosing the teaching strategy for the development of technical and transversal skills. To exemplifies the use of the referred framework in the choice of the active learning method to be used, the course Methodology of Scientific and Technological Research of a Production Engineering program was used, in a specific topics of his syllabus. The research methods used to develop this work were the case study and the survey. The latter was made necessary because the proposed framework has a survey as a step, in which students to identify their preferences in relation to active learning strategies.
\end{abstract}

Key-words: Active learning. Framework. Technical Skills. Soft skills. 